\title{
An application of composite complex sinusoidal modeling to the estimation of directions and waveforms of incident plane waves
}

\author{
Masato Abe, Jiaxiang Liu, and Ken'iti Kido \\ Research Center for Applied Information Sciences, Tohoku University, \\ 2-1-1, Katahira, Sendai, 980 Japan
}

(Received 21 August 1989)

\begin{abstract}
Composite complex sinusoidal modeling (CCSM) offered a better bearing characteristic than other methods using auto regressive modeling (ARM). However, neither method can estimate the waveforms. Therefore, a new CCSM to estimate both the directions and waveforms of incident sounds has been devised. From the results of computer simulation using 16 sensors, two sound sources with the same power in the $40^{\circ}$ and $75^{\circ}$ directions were separated, and the waveform of the sound from the $40^{\circ}$ direction was estimated to decrease by $34 \mathrm{~dB}$ the effect of the sound from the $75^{\circ}$ direction.
\end{abstract}

PACS number: 43. 30. Wi, 43. 50. Yw, 43. 60. Gk

\section{INTRODUCTION}

To achieve a better bearing characteristic, many methods such as auto regressive modeling (ARM) ${ }^{1-3}$ ) and composite complex sinusoidal modeling $(\mathrm{CCSM})^{4)}$ have been proposed to estimate both the directions and power spectra of incident plane waves using an equi-spaced sensor array. From the sensor outputs a spatial sequence is calculated for every frequency component. When there are many plane waves, the spatial sequence is expressed by the sum of the same number of complex sinusoidal waves as that of the incident plane waves. The direction and spectrum of each incident wave can be estimated from the phase change rate and the amplitude of the corresponding complex sinusoidal wave. CCSM gives better bearing characteristics than ARM, since it assumes that the sequence is expressed by a sum of complex sinusoidal waves while ARM assumes that the sequence is expressed by a sum of exponentially decaying complex sinusoidal waves. However, the waveform cannot be estimated either by CCSM or by ARM. Therefore, a new CCSM is described which estimates not only the directions but also the waveforms of incident plane waves. The effective- ness of the new CCSM is proved by a computer simulation.

\section{PRINCIPLE}

It is assumed that a plane wave comes from the direction $\theta$ to the uniformly spaced array composed of $N$ microphones named $M_{0}, M_{1}, \ldots, N_{N-1}$ as shown in Fig. 1. Then, the output of the microphone $M_{n}$ is given as

$$
x_{n}(t)=x_{0}(t-n d \cos \theta / c),
$$

where $x_{0}(t)$ denotes the output of the microphone $M_{0}, d$ the distance between two adjacent microphones and $c$ the sound velocity. The distance $d$ should be less than half the wavelength of the incident sound in order to avoid the effect of spatial aliasing, which may produce a ghost of sound source.

All of the microphone outputs are band-limited using appropriate analog low-pass filters, and A/D converted at a sampling rate of $f_{\mathrm{s}}$. Let the $m$-th sample of the output of the $n$-th microphone be $x_{n}{ }^{\prime}(m)$, then

$$
\begin{aligned}
x_{n}{ }^{\prime}(m) & =x_{n}\left(m / f_{\mathrm{s}}\right) \\
& =x_{0}\left(m / f_{\mathrm{s}}-n d \cos \theta / c\right) .
\end{aligned}
$$

The sequence $x_{n}{ }^{\prime}(m)$ is cut out by multiplying an 


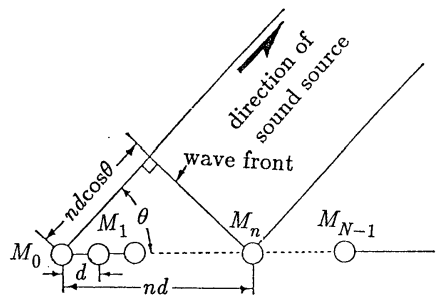

Fig. 1 Relation between an incident plane wave from $\theta$ direction and a linear array composed of equi-spaced $N$ microphones.

adequate time window $w(m)$ with the length $L$, and is separated into different frequency components by discrete Fourier transform (DFT). The $k$-th frequency component $X_{n}(k)$ is expressed as

$$
X_{n}(k)=\frac{1}{L} \sum_{m=0}^{L-1} w(m) x_{n}{ }^{\prime}(m) \exp (-j 2 \pi m k / L) .
$$

The observed time $T=L / f_{\mathrm{s}}$, corresponding to the length $L$ of the time window, should be sufficiently long so that the outputs of the microphones are highly correlated with one another: the duration $T$ of the time window should be longer than the period in which an incident sound propagates from one end of the array to the other. In this case, the spectra $X_{n}(k)$ of the sequence $w(m) x_{n}{ }^{\prime}(m)$, where $n=0,1,2, \ldots, N-1$, differ from one another only in phase. Hence, Eq. (3) is approximately expressed using a phase change rate (PCR) $z$ as follows:

$$
X_{n}(k) \approx X_{0}(k) z^{n}
$$

where

$$
z=\exp \left(-j 2 \pi \frac{k d}{T} \cos \theta / c\right) .
$$

A spatial sequence $Y_{k}(n)$ is obtained for each discrete frequency $k / T$ by arranging the $k$-th frequency components $X_{n}(k)$ according to the order of microphone number $n$ :

$$
\begin{aligned}
Y_{k}(n) & =X_{n}(k) \\
& \approx X_{0}(k) z^{n} .
\end{aligned}
$$

The spectrum $X_{0}(k)$ and the direction $\theta$ of the incident plane wave can be estimated by evaluating the amplitude and the PCR $z$ of the complex sinusoidal wave.

When several incident plane waves come from different directions, the spatial sequence is expressed by the sum of the same number of complex sinusoidal waves as that of the incident plane waves, the direction of which is estimated by evaluating the PCR $z$ of the corresponding complex sinusoidal wave. When $P$ plane waves come in, the spectrum $X_{n}(k)$ of the output of microphone $M_{n}$ is expressed as

$$
\begin{aligned}
X_{n}(k) & =\sum_{p=1}^{P} A_{p}(k) z_{p}{ }^{n} \\
& =\sum_{p=1}^{P} A_{p}(k) \exp \left(-j 2 \pi \frac{k}{T} n d \cos \theta_{p} / c\right),
\end{aligned}
$$

where $A_{p}(k)$ represents the spectrum of the output of microphone $M_{0}$ for the $p$-th plane wave with the incident angle $\theta_{p}$.

It is clear from Eq. (7) that the spatial sequence $Y_{k}(n)=X_{n}(k), n=0,1, \ldots, N-1$, is composed of $P$ complex sinusoidal waves with amplitudes $A_{p}(k)$ 's and PCR $z_{p}$ 's. Therefore, the spectra $A_{p}(k)$ 's and the directions $\theta_{p}$ 's of incident plane waves, where $p=1,2, \ldots, P$, can be estimated by evaluating the amplitude and the PCR of the corresponding complex sinusoidal wave.

The analysis by CCSM is based on the assumption that the spatial sequence to be estimated is composed of several complex sinusoidal waves, ${ }^{4)}$ whereas in ARM analysis it is assumed that the sequence is composed of several exponentially decaying complex sinusoidal waves, and the power of each sinusoidal wave is estimated approximately by all pole modeling. ${ }^{1-3)}$ Therefore, CCSM is more suitable than ARM for application to the array signal processing since the spatial sequence made of array outputs is represented by the sum of nondecaying complex sinusoidal waves. However, the waveforms cannot be estimated either by the CCSM or by ARM.

The new CCSM devised for waveform estimation is derived as follows. It is assumed that there are $I$ incident plane waves. Then, the spatial sequence $Y_{k}(n)$ for the $k$-th frequency component is expressed using Eq. (7):

$$
Y_{k}(n)=X_{n}(k)=\sum_{i=1}^{I} A_{i}(k) z_{i}{ }^{n},
$$

where

$$
z_{i}=\exp \left(-j 2 \pi \frac{k}{T} d \cos \theta_{i} / c\right), \quad i=1,2, \ldots, I .
$$

In Eq. (8), the spatial sequence $Y_{k}(n)$ is the function of $2 I$ parameters: $A_{i}(k)$ and $z_{i}$, where $i=1,2, \ldots, I$.

By solving the Eq. (8), both the spectra $A_{i}(k)$ 's and the PCR $z_{i}$ 's, $i=1,2, \ldots, I$, are estimated. The 


\section{ABE et al.: CCSM TO ESTIMATE DIRECTIONS AND WAVEFORMS OF SOUNDS}

algorithm to estimate the spectra $A_{i}(k)$ 's and the PCR $z_{i}$ 's is explained below.

First, introduced is a polynomial $B_{I}(z)$ of order $I$ which falls to 0 at $z=z_{i}$ as follows:

$$
B_{I}(z)=\sum_{q=0}^{I} b_{q} z^{q}=\prod_{i=1}^{I}\left(z-z_{i}\right),
$$

where $b_{I}=1$. Multiplying both sides of Eq. (8) by $B_{I}\left(z_{i}\right)$, the following equations are obtained:

$$
\begin{aligned}
X_{n}(k) B_{I}\left(z_{i}\right) & =\sum_{i=1}^{I} A_{i}(k) z_{i}{ }^{n} B_{I}\left(z_{i}\right) \\
& =\sum_{i=1}^{I} A_{i}(k) \sum_{q=0}^{I} b_{q} z_{i}{ }^{q+n} \\
& =\sum_{q=0}^{I} b_{q} \sum_{i=1}^{I} A_{i}(k) z_{i}{ }^{q+n} \\
& \approx \sum_{q=0}^{I} b_{q} X_{q+n}(k) .
\end{aligned}
$$

The following equation is obtained from Eq. (11) because $B_{I}\left(z_{i}\right)=0$ and $b_{I}=1$ :

$$
\begin{gathered}
\sum_{q=0}^{I-1} b_{q} X_{q+n}(k) \approx-X_{I+n}(k), \\
n=0,1, \ldots, 2 I-1 .
\end{gathered}
$$

In Eq. (12), $b_{q}$ 's, where $q=0,1, \ldots, I-1$, are unknown; the number of unknown parameters is $I$. However, there are $2 I$ equations in (12). Therefore, $b_{q}$ 's which satisfy all equations in (12) cannot always be obtained. Thus, $b_{q}$ 's are calculated approximately by solving the first $I$ equations, that is, for $n=0,1, \ldots, I-1$ in Eq. (12).

Next, $z_{i}$ 's are calculated by solving the following polynomial, whose coefficients $b_{q}$ 's are obtained approximately by Eq. (12).

$$
B_{I}(z)=z^{I}+b_{I-1} z^{I-1}+\ldots+b_{1} z+b_{0}=0
$$

Since $b_{q}$ 's are the approximate values, $z_{i}$ 's obtained by Eq. (13) have some errors. Therefore, the estimated $z_{i}$ 's are modified or deleted by the following criteria. ${ }^{4}$

$$
\begin{gathered}
\text { (a) if } \quad\left|\angle z_{i}\right|>2 \pi \frac{k d}{T} / c+\Delta \phi, \quad z_{i} \text { is deleted, } \\
\text { (b) if } \quad 2 \pi \frac{k d}{T} / c \leq \angle z_{i} \leq 2 \pi \frac{k d}{T} / c+\Delta \phi, \\
\quad \tilde{z}_{i}=\exp \left(j 2 \pi \frac{k d}{T} / c\right) \\
\text { (c) if } \quad-2 \pi \frac{k d}{T} / c-\Delta \phi \leq \angle z_{i} \leq-2 \pi \frac{k d}{T} / c \\
\tilde{z}_{i}=\exp \left(-j 2 \pi \frac{k d}{T} / c\right)
\end{gathered}
$$

(d) else $\quad \tilde{z}_{i}=\frac{z_{i}}{\left|z_{i}\right|}$,

where $\Delta \phi$ is a small positive value, and was set to $2 \pi(k d / T) / 10 c$ in the computer simulation expressed in section 3. The resultant number $\tilde{I}$ of sound source and the resultant phase change rates $\tilde{z}_{i}$ 's, $i=0,1, \ldots, \tilde{I}-1$, are determined for the assumed number $I$.

Finally, the directions $\theta_{i}$ 's are estimated from the PCR $\tilde{z}_{i}$ 's by Eq. (5), and the spectra $A_{i}(k)$ 's are estimated by solving the following equation:

$$
X_{n}(k)=\sum_{i=1}^{\tilde{I}} A_{i}(k) \tilde{z}_{i}{ }^{n},
$$

The above processing is carried out for $I=1,2$, $\ldots, Q$, where $Q$ is the assumed maximum number of incident plane waves. The spectrum $A_{i}(k)$ and the direction $\theta_{i}$ of the $i$-th incident plane wave are estimated using the number $\tilde{I}$ obtained when the following prediction error reaches the smallest value.

$$
\boldsymbol{P}_{I, \tilde{I}}=\sum_{n=0}^{N-1} \sum_{i=1}^{\tilde{I}}\left|A_{i}(k) \tilde{z}_{i}{ }^{n}-X_{n}(k)\right|^{2} .
$$

After executing the above mentioned processing for every frequency component, the waveform from a specified direction can be estimated by gathering the spectrum $A_{i}(k)$ from the specified direction, and calculating the inverse Fourier transform.

\section{COMPUTER SIMULATION}

A linear array of microphones, of length $2 \mathrm{~m}$, composed of 16 sensors was arranged as shown in Fig. 2. There were two incident sound sources in the $40^{\circ}$ and $75^{\circ}$ directions, radiating white noise with the same power, and band-limited between $80 \mathrm{~Hz}$ and $1.2 \mathrm{kHz}$. The sampling frequency was $10 \mathrm{kHz}$, and the length of time window $L$ was 1,024 . The waveform of the sound in the $40^{\circ}$ direction was reconstructed from the components whose incident angles were estimated to be between $35^{\circ}$ and $45^{\circ}$.

Figure 3 shows the original power spectrum of the output of the microphone $M_{0}$ and the error spectrum, which is the absolute value of the difference between the original spectrum and the reconstructed one. The normalized mean square error between the real waveform and the reconstructed one was $-34 \mathrm{~dB}$. That is, the effect of incident sound from the $75^{\circ}$ direction was suppressed by $34 \mathrm{~dB}$. This means that a waveform can be estimated correctly 


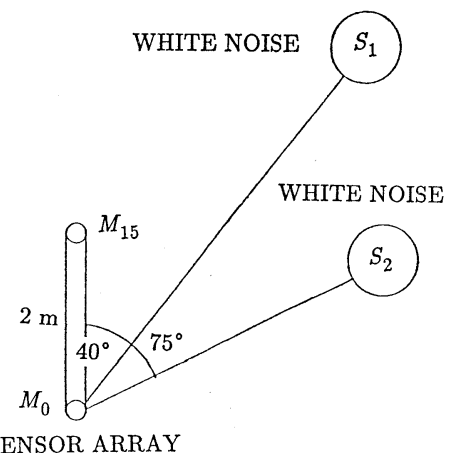

Fig. 2 Arrangement of two incident plane waves and a linear array of microphones. Sampling frequency: $10 \mathrm{kHz}$. Length of time window $L=1,024$. Length of the array: $2 \mathrm{~m}$. Number of microphones: 16 . Two incident plane waves radiate white noise band-limited between $80 \mathrm{~Hz}$ and 1.2 $\mathrm{kHz}$.

by the newly proposed CCSM.

\section{CONCLUSION}

A new method is proposed to estimate not only directions but also the waveforms of incident plane waves with a high bearing resolution. In a computer simulation using two sound sources at $40^{\circ}$ and $75^{\circ}$, the waveform of the sound from the $40^{\circ}$ was estimated, using 16 sensors to decrease by $34 \mathrm{~dB}$ the effect of the sound from the $75^{\circ}$ direction.

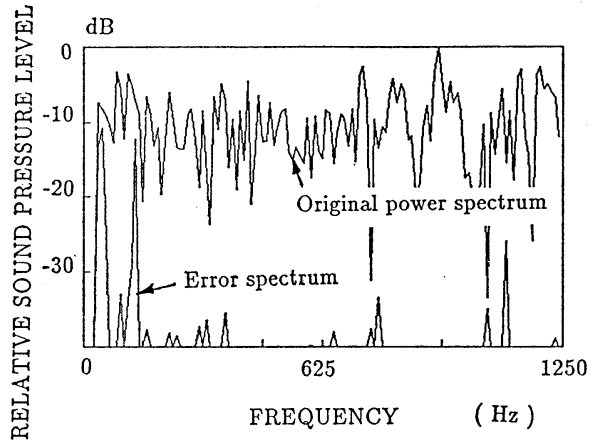

Fig. 3 Original power spectrum of the incident plane wave in $40^{\circ}$ direction and error spectrum which is the difference between the original spectrum and the reconstructed one.

\section{REFERENCES}

1) H. P. Bucker, "High resolution cross-sensor beamforming for a uniform line array," J. Acoust. Soc. Am. 63, 420-424 (1978).

2) D.P. Skinner, S.M. Hedlicka, and A.D. Matthews, "Maximum entropy array processing," J. Acoust. Soc. Am. 66, 488-493 (1979).

3) H. P. Bucker, "Cross-sensor beamforming with a sparse line array," J. Acoust. Soc. Am. 61, 494498 (1977).

4) M. Abe and K. Kido, "An application of composite complex sinusoidal modeling to the estimation of direction and spectra of an incident plane wave," J. Acoust. Soc. Jpn. (E) 3, 213-223 (1982). 